\title{
Evaluation of Coronary Stents In Vitro With CT Angiography Effect of Stent Diameter, Convolution Kernel, and Vessel Orientation to the Z-Axis
}

\author{
Shigeru Suzuki, MD; Shigeru Furui, MD; Tatsuro Kaminaga, MD; Teiyu Yamauchi, MD; \\ Sadatoshi Kuwahara, RT*; Naoyuki Yokoyama, MD**; \\ Masatoshi Suzuki, MD**; Takaaki Isshiki, MD**
}

\begin{abstract}
Background The aims of the present study were to assess the effect of the stent diameter, convolution kernel, and vessel orientation to the z-axis on the evaluation of coronary stents, in vitro with computed tomography (CT) angiography.

Methods and Results Seven vascular models (2 models without stenosis, 2 with obstruction, and 3 with stenosis) with an approximate inner diameter of 3 or $4 \mathrm{~mm}$, filled with contrast material ( 79 or $330 \mathrm{HU}$ ) were scanned with a 16-detector CT. The diameter measurement of the stent lumen and stenosis evaluation were both done in an orientation parallel to the z-axis of the scanner using 4 convolution kernels. The measured diameters of the stented lumen were 47-57\% and 36-45\% smaller than the actual inner diameter of the 3- and 4-mm diameter models, respectively. The diameter measurement of the stent lumen and visualization of the in-stent stenosis were improved by using convolution kernels with higher spatial resolution. The in-stent artifacts were evaluated in 4 orientations $\left(0^{\circ}, 30^{\circ}, 60^{\circ}, 90^{\circ}\right)$ to the z-axis. The artifact was the minimum in $0^{\circ}$ to the z-axis, and the maximum in $90^{\circ}$.

Conclusion Visualization of the lumen of a stent by CT is affected by its diameter, convolution kernel, and vessel orientation to the z-axis, and these factors should be taken into consideration in the stent evaluation. (Circ J 2005; 69: 1124-1131)
\end{abstract}

Key Words: Convolution kernel; Coronary stent; CT angiography

C oronary angiography with multidetector computed tomography (CT) has been developed and begun to be used clinically!,2 The stent evaluation in one of the most important issues for CT angiography. Investigators have evaluated coronary stents using electron beam ${ }^{3-6}$ or multidetector $\mathrm{CT}^{7-11}$ and the effects of the convolution kernel, the number of detector rows of the CT scanner, and the patient's heart rate on the lumen visibility or CT attenuation inside the stent have been estimated. However, few reports are available on the effects of the stent diameter and vessel orientation to the $\mathrm{z}$-axis.

We used CT angiography to assess the effect of stent diameter, convolution kernel, and vessel orientation to the $\mathrm{z}$-axis on the evaluation of coronary stents, in vitro, with analyses using CT attenuation profiles. To our knowledge, this is the first such study of these factors.

\footnotetext{
Methods

Phantom Design (Fig 1)

We made 7 vascular models, 2 without stenosis, 2 with obstruction, and 2 with stenosis, using tubes made of ethyl-

(Received February 10, 2005; revised manuscript received May 30, 2005; accepted June 2, 2005)

Department of Radiology, Teikyo University School of Medicine, *CT Clinical Science, Philips Medical Systems and **Department of Medicine, Teikyo University School of Medicine, Tokyo, Japan

Mailing address: Shigeru Suzuki, MD, Department of Radiology, Teikyo University School of Medicine, 2-11-1 Kaga, Itabashi-ku, Tokyo 173-8605, Japan
}

ene-vinyl alcohol copolymer (Soanor; Nippon Synthetic Chemical Industry Co, Ltd, Tokyo, Japan) with an approximate inner diameter of 3 or $4 \mathrm{~mm}$ and $0.5-\mathrm{mm}$ thickness. The tubes were soaked in water for 7 days before use. The inner diameter of each tube was measured 10 times by a profile projector (Nikon V-12B; Nikon Co, Tokyo, Japan) to the nearest 100th of a millimeter. The average inner diameter was $3.16 \mathrm{~mm}$ for the $3-\mathrm{mm}$ diameter model and $4.07 \mathrm{~mm}$ for the $4-\mathrm{mm}$ diameter model. The CT attenuation of the tube wall was $76 \pm 8 \mathrm{HU}($ mean $\pm \mathrm{SD})$.

Balloon-expandable stents made from stainless steel wire with a strut diameter of $0.14-\mathrm{mm}$ and a length of $18-\mathrm{mm}$ (Bx-Velocity; Johnson \& Johnson, Miami Lakes, FL, USA) were used. Stents with a nominal diameter of 3 or $4 \mathrm{~mm}$ were implanted in the mid portion of tubes with a corresponding inner diameter.

To create the vascular models without stenosis $(0 \%$ stenosis model), stent-mounted tubes with a 3- and 4-mm inner diameter were filled with contrast material (iohexiol) diluted to $330 \pm 7 \mathrm{HU}$ and closed at both ends (Fig 1a,c).

Vessel obstruction was simulated by filling the tubes with diluted contrast material $(79 \pm 9 \mathrm{HU})$ to approximate the CT attenuation of the tube wall. The 2 vascular models with obstruction (100\% stenosis model) were made by filling the stent-mounted tubes with a 3- and 4-mm inner diameter with contrast material diluted to $79 \mathrm{HU}$.

We made 3 vascular models with stenosis as follows (Fig 1a,d). Type 1 (50\% diameter reduction model): a tube with a 2-mm inner diameter, was filled with contrast material diluted to $330 \mathrm{HU}$ and then fixed within a stent-mounted 

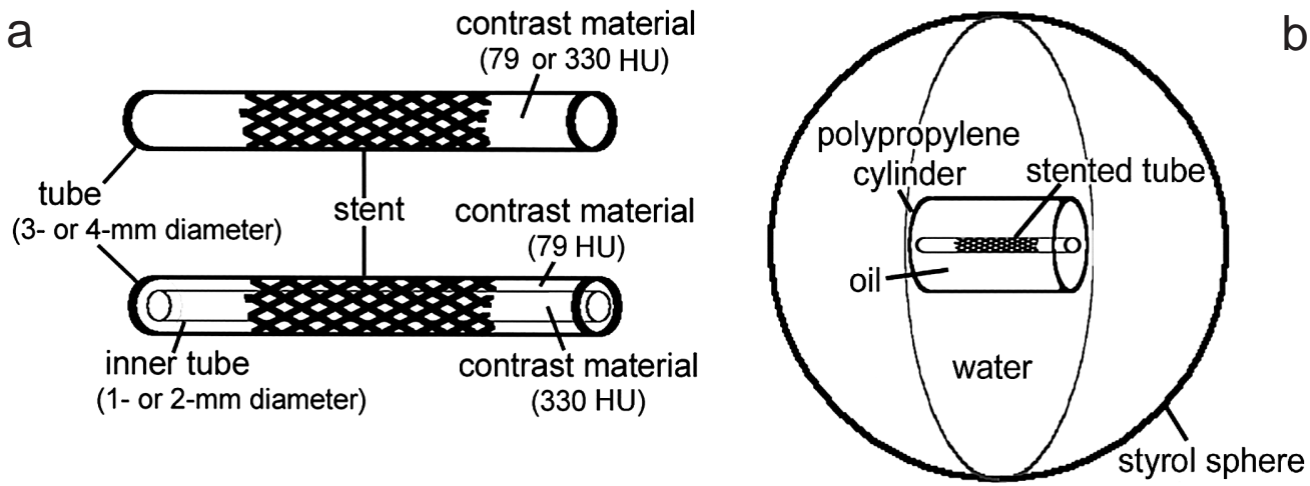

C
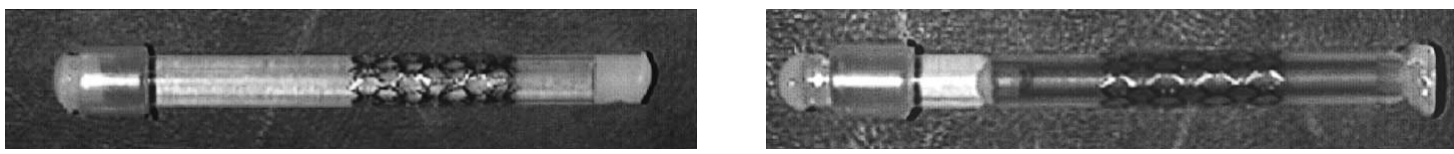

e
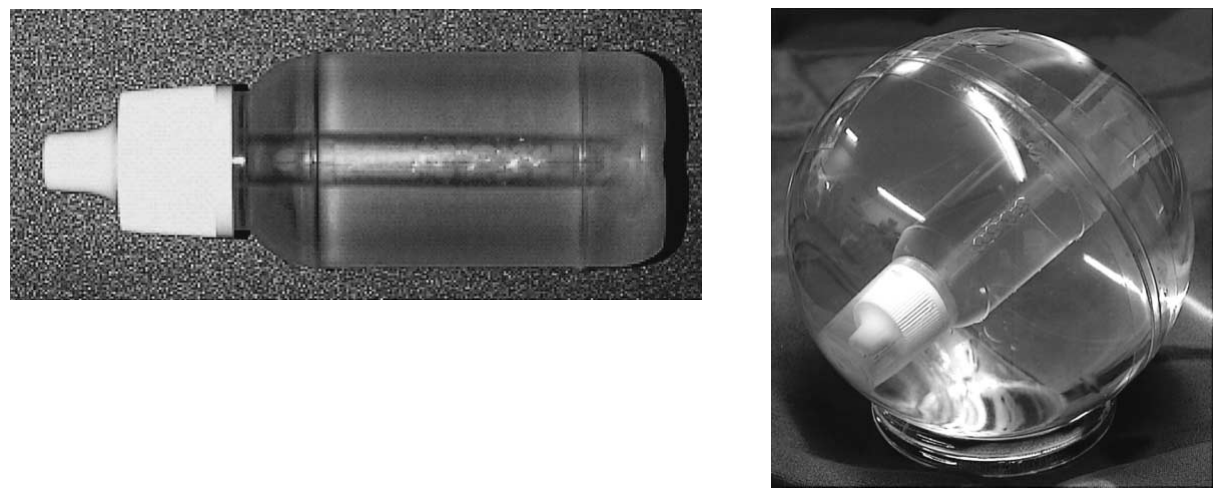

Fig 1. (a) Bx-Velocity stent was implanted into the tube with an inner diameter of 3 or $4 \mathrm{~mm}$. Contrast material diluted to $330 \mathrm{HU}$ and $79 \mathrm{HU}$ was used to fill the stent-mounted tubes as $0 \%$ and $100 \%$ stenosis models, respectively (Upper model). In the 50\% and 75\% stenosis models, a smaller tube filled with contrast material diluted to $330 \mathrm{HU}$ was fixed in a stent-mounted tube that was filled with contrast material diluted to $79 \mathrm{HU}$ (Lower model). (b) Vascular model fixed in a polypropylene cylinder filled with salad oil and aligned parallel with the central axis of cylinder. The polypropylene cylinder is fixed in a water-filled spherical styrol container, with the central axes overlapping. (c) Vascular model without stenosis. (d) Vascular model with stenosis. (e) Vascular model in the polypropylene cylinder filled with salad oil. (f) Spherical phantom including the vascular model.

tube with a 4-mm inner diameter filled with contrast material diluted to $79 \mathrm{HU}$. Type 2 (75\% diameter reduction model): a tube with an inner diameter of 1-mm filled with contrast material diluted to $330 \mathrm{HU}$ was fixed within a stent-mounted tube with a 4-mm inner diameter in the same manner as for Type 1. Type 3 (67\% diameter reduction model): a 1-mm inner diameter tube was fixed within a stent-mounted tube with a 3-mm inner diameter using the same procedure as for Types 1 and 2. In these models, we considered the wall of the inner tube and the contrast material of $79 \mathrm{HU}$ as corresponding to neointima.

We fixed each vascular model in a polypropylene cylinder (diameter $\approx 2.6 \mathrm{~cm}$ ) filled with salad oil (CT attenuation, $-120 \pm 4 \mathrm{HU})$, locating the vascular model parallel with the central axis of the polypropylene cylinder (Fig 1e). We fixed the polypropylene container in a water-filled styrol spherical container (diameter $\approx 12 \mathrm{~cm}$ ), with their central axes overlapping (Fig 1b,f).

\section{CT Scanning}

We placed the phantom in the scanner gantry, setting the center of the spherical container of the phantom on the axis of the gantry rotation. All CT scans were performed with a 16-detector CT scanner (MX 8000 IDT, Philips Medical Systems, Cleveland, OH, USA), using a retrospective electrocardiogram (ECG) gating technique. An artificial ECG of 60 beats/min was recorded by an ECG Simulator. Parameters were $16 \times 0.75-\mathrm{mm}$ collimation, $5.7-\mathrm{mm} / \mathrm{s}$ table speed, 0.42-s per gantry rotation, 200-mAs tube current, and $120 \mathrm{kV}$ tube voltage. The scan FOV was $50 \mathrm{~cm}$ in diameter.

The raw data were reconstructed in $18-\mathrm{cm}$ FOV, $512 \times$ 512 pixel matrix, and $0.8-\mathrm{mm}$ thickness at $0.4-\mathrm{mm}$ intervals, using 4 convolution kernels: CA (smooth), CB (medium), CC (detail), and CD (bone). According to the information provided by Philips Medical Systems, contrast resolution tends to decrease in the following order: CA, $\mathrm{CB}, \mathrm{CC}, \mathrm{CD}$. The reconstructed images were transferred to a workstation (Virtual Place Advance, Version 2.0113; AZE Co, Ltd, Tokyo, Japan).

\section{Measurement of Stent Lumen Diameter}

The vascular models were positioned parallel to the $\mathrm{z}$ axis of the scanner. We used 10 consecutive axial images reconstructed in $0.8-\mathrm{mm}$ thickness at $0.4-\mathrm{mm}$ intervals by the 4 convolution kernels of the $0 \%$ stenosis models with a 

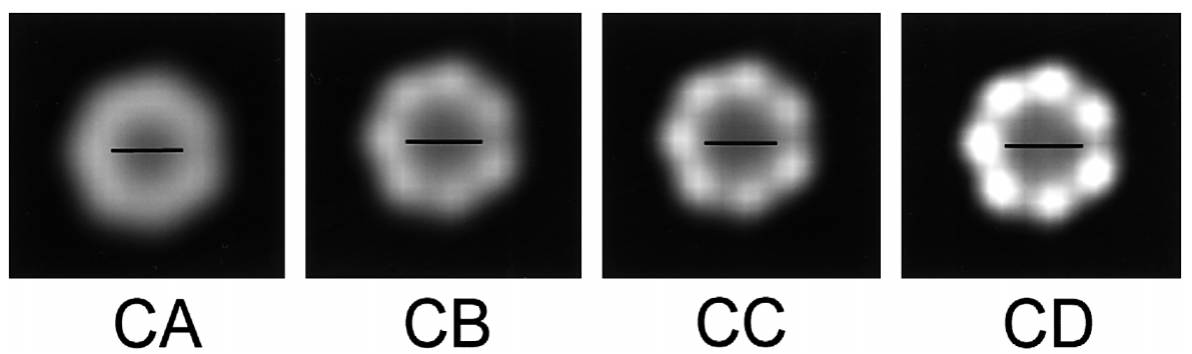

Fig 2. Axial images of the $0 \%$ stenosis models with a 4-mm inner diameter reconstructed by the 4 convolution kernels in a window width of $1,000 \mathrm{HU}$ and a center of $400 \mathrm{HU}$. Straight lines on the images correspond to diameters measured manually using electronic calipers. Images from left to right indicate $\mathrm{CA}, \mathrm{CB}, \mathrm{CC}$, and CD.

Table 1 Measurement of Stent Lumen Diameter

\begin{tabular}{ccccc}
\hline \hline \multirow{2}{*}{ Stent diameter } & \multicolumn{4}{c}{ Convolution kernel } \\
\cline { 2 - 5 } & $C A$ & $C B$ & $C C$ & $C D$ \\
\hline $3 \mathrm{~mm}$ & $-1.79 \pm 0.15$ & $-1.58 \pm 0.15$ & $-1.47 \pm 0.13$ & $-1.49 \pm 0.14$ \\
$4 \mathrm{~mm}$ & $-1.84 \pm 0.21$ & $-1.63 \pm 0.13$ & $-1.46 \pm 0.16$ & $-1.52 \pm 0.15$ \\
\hline
\end{tabular}

The difference of the inner diameter of the stent as measured on the computed tomography image minus the actual inner diameter as measured on the model is given as: mean \pm standard deviation $(\mathrm{mm})$. There was no significant difference in the measurement errors between the 2 inner diameters. There were significant differences in the measurement errors between $C A$ and $C B(p=0.0001)$, $C B$ and $C C(p=0.0111)$, but no significant difference in the measurement errors between $C B$ and $C D$, or $C D$ and $C C$.

3- and 4-mm inner diameter in a window width of 1,000 HU and a center of $400 \mathrm{HU}$. The diameter of the stent lumen was measured manually using electronic calipers measuring to $0.1 \mathrm{~mm}$ (Fig 2). A total of 10 separate diameter measurements were performed on the 10 consecutive axial images in each vascular model. The measurement was done on the line that was parallel with the $\mathrm{x}$-axis on each image. The measurement error was defined as the difference of the inner diameter of the stent as measured on the CT image minus the actual inner diameter as measured on the model.

Statistical analysis involved two-way analysis of variance with repeated measures to assess interactions among combinations of the 4 convolution kernels and 2 inner diameters of the vascular models. To compare parameters, Scheffe tests were used as the post tests following the analyses of variance with repeated measures. A p-value $<0.05$ was considered to represent a statistically significant result.

\section{Stenosis Evaluation}

The vascular models were positioned parallel to the $\mathrm{z}$-axis of the scanner. Axial images reconstructed by the 4 convolution kernels and longitudinal reformations, including the centerline of all of the 7 vascular models, were evaluated in a window width of $1,000 \mathrm{HU}$ and a center of $400 \mathrm{HU}$, and compared with the unstented portion of the model without stenosis. To evaluate the visibility of in-stent stenosis, stents images were evaluated in random order by one blinded experienced radiologist and 2 radiology technicians in consensus. The in-stent stenosis was evaluated with a 3-point scale, based on clinical significance considering the indication and the degree of difficulty of percutaneous coronary intervention: $1=$ no significant stenosis (diameter reduction $<50 \%$ ) visible, $2=$ significant stenosis (diameter reduction $\geq 50 \%$ ) visible, $3=$ obstruction visible.

\section{Effect of Vessel Orientation to the Z-Axis}

In the evaluation of the effect of vessel orientation, the vascular models were positioned in 4 orientations $\left(0^{\circ}, 30^{\circ}\right.$, $60^{\circ}, 90^{\circ}$ ) to the $\mathrm{z}$-axis of the scanner on the horizontal plane. We used CD as the convolution kernel. The horizontal and vertical longitudinal reformations, including the center line of the 4-mm diameter model without stenosis in the 4 orientations, were evaluated in a window width of $1,000 \mathrm{HU}$ and a center of $400 \mathrm{HU}$. The thickness of reformation images was equal to the size of 1 boxel.

To evaluate artifacts inside the stents, the stent images were evaluated in random order by one blinded experienced radiologist and 2 radiology technicians in consensus. The in-stent artifact was evaluated with a 3-point scale: $1=$ mild artifacts, $2=$ moderate artifacts with degradation of image quality, $3=$ severe artifacts, vicinity of the stent not assessable.

In addition, the attenuation values inside the visible stent lumen were measured with a region-of-interest (ROI) technique. The size of the rectangular ROI was $1.5 \times 12 \mathrm{~mm}$. To evaluate the artifacts inside the stents, the standard deviations of the luminal CT attenuation were evaluated with F-test; $p<0.05$ was considered to represent a statistically significant result.

\section{Results}

\section{Measurement of Stent Lumen Diameter}

No interactions were found among any combinations of the factors $(p=0.8128)$. There were significant differences $(p<0.0001)$ in the measurement errors among the 4 convolution kernels, but no significant difference $(p=0.653)$ in the measurement errors between the 2 inner diameters of the vascular models.

The inner diameters of the vascular models were underestimated by CT angiography with mean measurement errors of -1.46 to $-1.84 \mathrm{~mm}$. The absolute mean overall measurement error decreased in the following order: $\mathrm{CA}$, $\mathrm{CB}, \mathrm{CD}, \mathrm{CC}$ (Table 1). There were significant differences in the measurement errors between $\mathrm{CA}$ and $\mathrm{CB}(\mathrm{p}=0.0001)$, $\mathrm{CB}$ and $\mathrm{CC}(\mathrm{p}=0.0111)$, but no significant difference in the measurement errors between $\mathrm{CB}$ and $\mathrm{CD}(\mathrm{p}=0.1009)$, or 
Table 2 Visibility of In-Stent Stenosis

\begin{tabular}{cllll}
\hline \hline \multirow{2}{*}{$\begin{array}{c}\text { Degree of stenosis/ } \\
\text { stent diameter }\end{array}$} & \multicolumn{4}{c}{ Convolution kernel } \\
\cline { 2 - 4 } & CA & $C B$ & $C C$ & CD \\
\hline $0 \% / 3 \mathrm{~mm}$ & No stenosis & No stenosis & No stenosis & No stenosis \\
$67 \% / 3 \mathrm{~mm}$ & Stenosis & No stenosis & Obstruction & Stenosis \\
$100 \% / 3 \mathrm{~mm}$ & Stenosis & Obstruction & Obstruction & Obstruction \\
$0 \% / 4 \mathrm{~mm}$ & No stenosis & No stenosis & No stenosis & No stenosis \\
$50 \% / 4 \mathrm{~mm}$ & No stenosis & No stenosis & Stenosis & Stenosis \\
$75 \% / 4 \mathrm{~mm}$ & Obstruction & Obstruction & Stenosis & Stenosis \\
$100 \% / 4 \mathrm{~mm}$ & Obstruction & Obstruction & Obstruction & Stenosis \\
\hline
\end{tabular}

The in-stent stenosis was evaluated with a 3-point scale: no significant stenosis (diameter reduction $<50 \%$ ) visible, significant stenosis (diameter reduction $\geq 50 \%$ ) visible, obstruction visible.
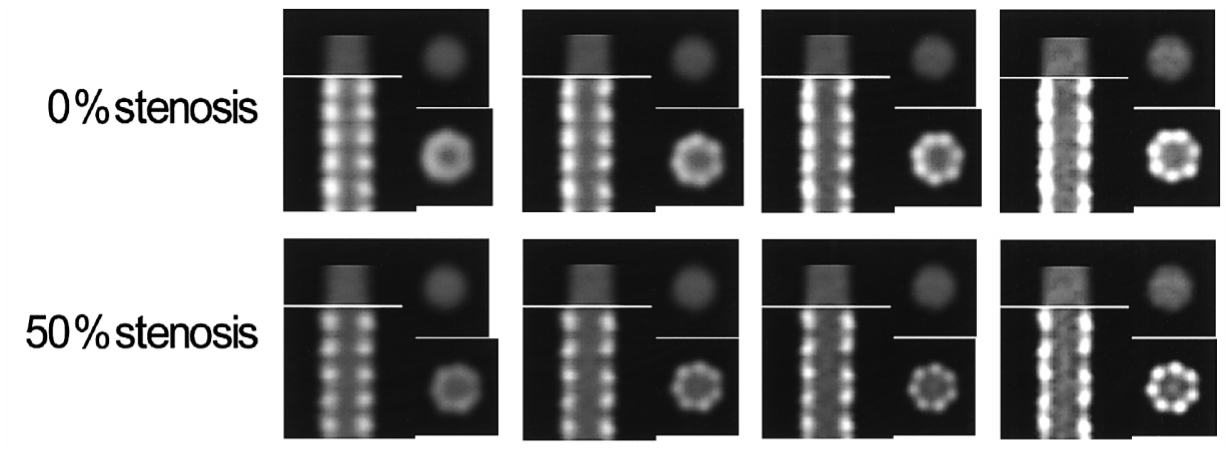

$75 \%$ stenosis
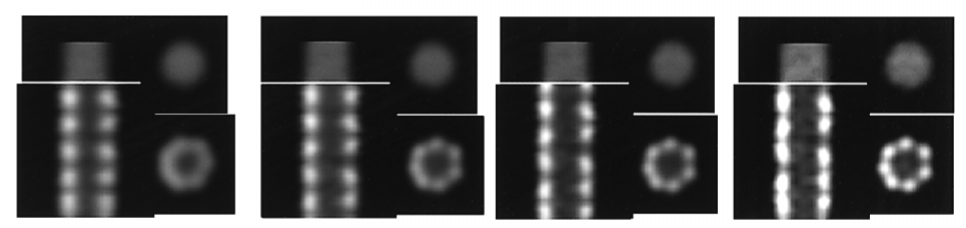

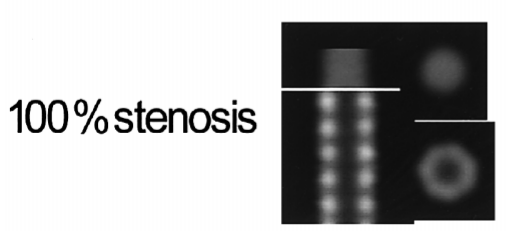

CA

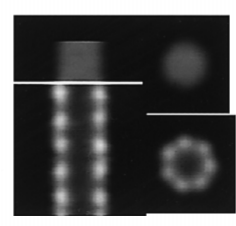

CB

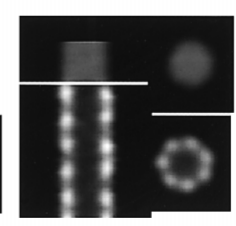

CC

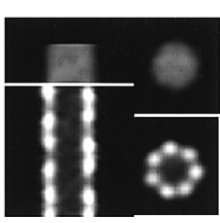

$\mathrm{CD}$

Fig 3. Axial images and longitudinal reformations of the 4-mm diameter models. Model without stenosis (First row), model with 50\% stenosis (Second row), model with 75\% stenosis (Third row), and model with obstruction (Fourth row). Four convolution kernels were used. Columns from left to right indicate $\mathrm{CA}, \mathrm{CB}, \mathrm{CC}$, and $\mathrm{CD}$.

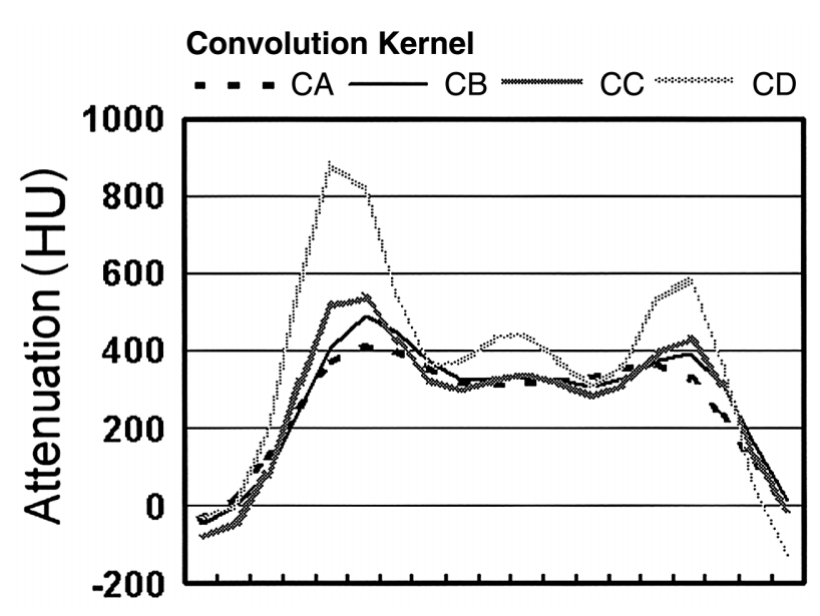

Fig 4. CT attenuation profile on cross-sections of the $50 \%$ stenosis models with 4-mm diameter using the 4 convolution kernels. Profile curve passes through the center of the vascular model. Increments of attenuation profile curve are 1 pixel.

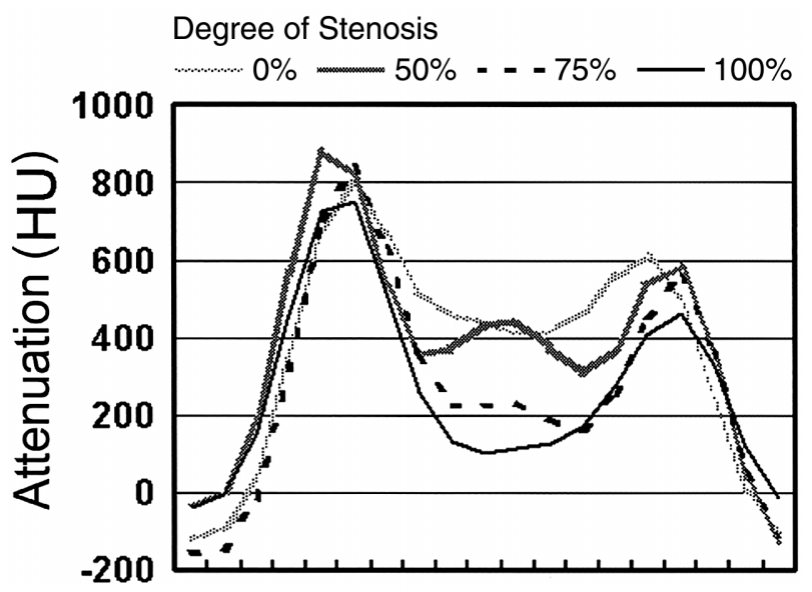

Fig 5. CT attenuation profile on cross-sections of the 4 models with 4-mm diameter using CD as the convolution kernel. Profile curve passes through the center of the vascular model. Increments of attenuation profile curve are 1 pixel. 


\section{horizontal}
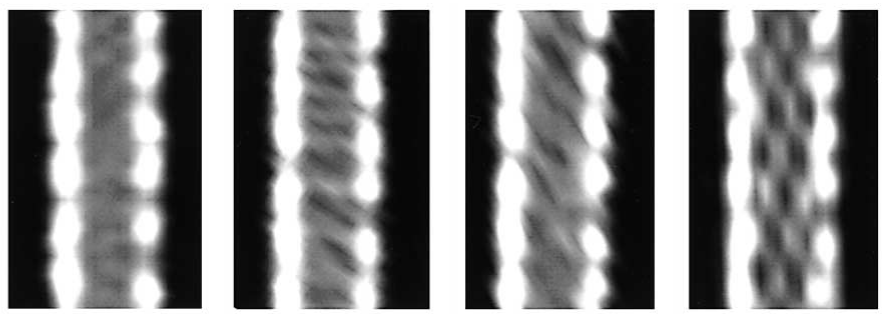

vertical
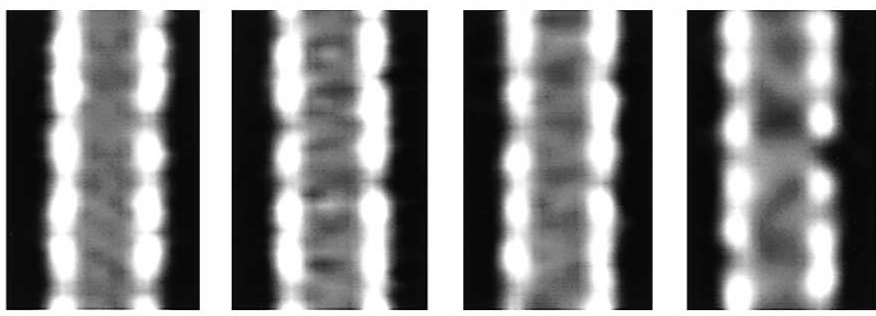

cross

section

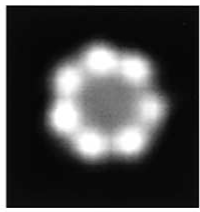

$0^{0}$

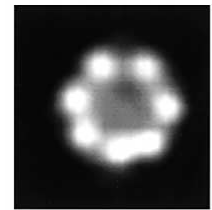

$30^{\circ}$

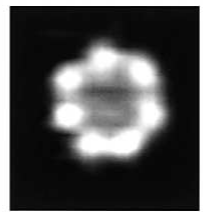

$60^{\circ}$

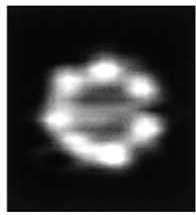

$90^{\circ}$

Fig 6. Longitudinal reformation and cross-section of the $0 \%$ stenosis model with 4-mm diameter. Horizontal longitudinal reformation (Upper row), vertical longitudinal reformation (Middle row), and cross-section (Lower row). Columns from left to right indicate $0^{\circ}, 30^{\circ}, 60^{\circ}$, and $90^{\circ}$ to the $\mathrm{z}$-axis of the scanner.

horizontal

vertical
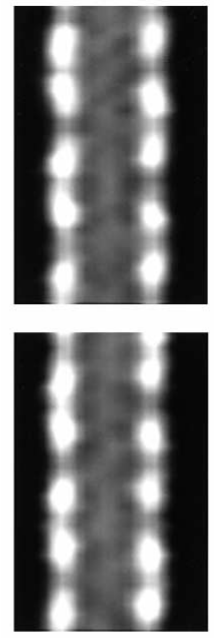

cross

section

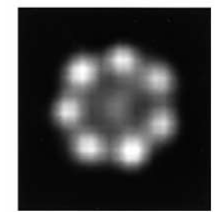

$0^{\circ}$
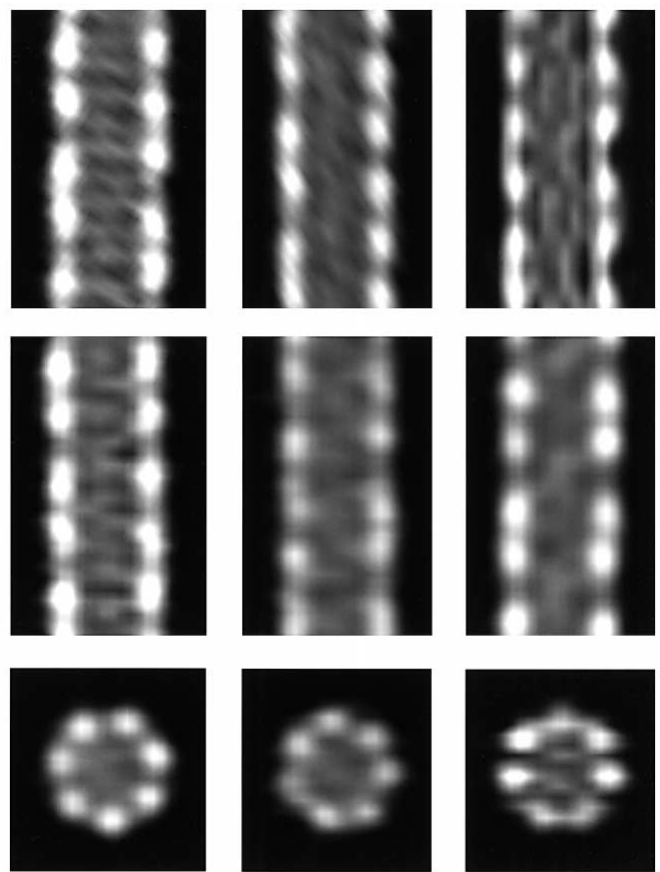

$60^{\circ}$

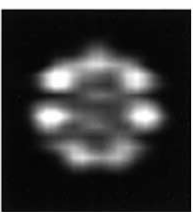

$90^{\circ}$

Fig 7. Longitudinal reformation and cross-section of the 50\% stenosis model with 4-mm diameter. Horizontal longitudinal reformation (Upper row), vertical longitudinal reformation (Middle row), and cross section (Lower row). Columns from left to right indicate $0^{\circ}, 30^{\circ}, 60^{\circ}$, and $90^{\circ}$ to the z-axis of the scanner. 
Table 3 Effect of Vessel Orientation to Z-Axis on Computed Tomography (CT) Attenuation and Artifacts Inside the Stents in the $0 \%$ Stenosis Model With 4-mm Diameter

\begin{tabular}{|c|c|c|c|c|}
\hline \multirow{2}{*}{ Direction of reformation } & \multicolumn{4}{|c|}{ Orientation to z-axis of the scanner } \\
\hline & $0^{\circ}$ & $30^{\circ}$ & $60^{\circ}$ & $90^{\circ}$ \\
\hline \multicolumn{5}{|l|}{ Horizontal } \\
\hline CT attenuation $(H U)$ & $432.0 \pm 32.0$ & $431.0 \pm 73.2$ & $430.2 \pm 72.0$ & $408.0 \pm 105.3$ \\
\hline Score & 1 & 2 & 2 & 3 \\
\hline \multicolumn{5}{|l|}{ Vertical } \\
\hline CT attenuation $(H U)$ & $427.7 \pm 37.2$ & $424.5 \pm 68.6$ & $427.7 \pm 66.0$ & $400.2 \pm 118.3$ \\
\hline Score & 1 & 2 & 2 & 3 \\
\hline
\end{tabular}

The CT attenuation of the stent lumen is given as: mean \pm standard deviation (HU). In-stent artifact was evaluated with a 3-point scale: $1=$ mild artifacts, $2=$ moderate artifacts with degradation of image quality, $3=$ severe artifacts, vicinity of the stent not assessable. In each direction of the reformation, the standard deviation of the luminal CT attenuation increased in the following order: $0^{\circ} ; 30^{\circ}$ and $60^{\circ} ; 90^{\circ}$ to the z-axis $(p<0.001)$. In the same orientation, there was no significant difference to the standard deviations between the horizontal and vertical reformations.

$\mathrm{CD}$ and $\mathrm{CC}(\mathrm{p}=0.7778)$. The measured diameters of the stented lumen were $47-57 \%$ and $36-45 \%$ smaller than the actual inner diameter of the 3- and 4-mm diameter models, respectively.

\section{Stenosis Evaluation}

Fig 2 shows the axial images reconstructed by the 4 convolution kernels and their longitudinal reformations of the 4-mm diameter models. Results of the stenosis evaluation for all vascular models by the 3 readers are summarized in Table 2. As shown in Fig 3 and Table2, it was difficult to recognize the difference between the 4-mm diameter models with $0 \%$ stenosis and 50\% stenosis using $\mathrm{CA}$ or $\mathrm{CB}$ as the convolution kernel. The visualization of the stent lumen was improved by using CC or CD. On the profile curve of the cross-section of the $50 \%$ stenosis model, there was a peak, corresponding to the patent lumen between the high attenuation of the stent struts in $\mathrm{CC}$ and CD (Fig 4). In contrast, there was no such peak with CA or $\mathrm{CB}$. The slopes of the profile curve corresponding to the boundary of the stent and the lumen were steeper in the following order: $\mathrm{CA}, \mathrm{CB}, \mathrm{CC}, \mathrm{CD}$. On the profile curves of the 4 models with a 4-mm diameter reconstructed $\mathrm{CD}$ (Fig 5), the peak corresponding to the patent lumen was smaller in the $75 \%$ stenosis model than in the $50 \%$ stenosis model. On the other hand, the portions with minimal CT attenuation, corresponding to the neointima, were approximately $100 \mathrm{HU}, 200 \mathrm{HU}$, and $300 \mathrm{HU}$ on the profile curves of the $100 \%, 75 \%$, and $50 \%$ stenosis models, respectively.

\section{Effect of Vessel Orientation to the Z-Axis}

Figs 6 and 7 showed the cross-sections and the horizontal and vertical longitudinal reformations of the $0 \%$ and $50 \%$ stenosis models of a $4-\mathrm{mm}$ diameter in the 4 orientations $\left(0^{\circ}, 30^{\circ}, 60^{\circ}, 90^{\circ}\right)$ to the $\mathrm{z}$-axis of the scanner. In the case of $30^{\circ}, 60^{\circ}$ or $90^{\circ}$ to the z-axis, it was difficult to recognize the difference between the $0 \%$ and $50 \%$ stenosis models because of the artifacts inside the stent. The effects of the vessel orientation on the CT attenuation and the artifacts inside the stents of the $0 \%$ stenosis models are summarized in Table 3 . In each direction of the reformation, the standard deviation of the luminal CT attenuation increased in the following order: $0^{\circ}, 30^{\circ}$ and $60^{\circ}$, then $90^{\circ}$ to the $\mathrm{z}$-axis $(\mathrm{p}<0.001)$. The standard deviation in $90^{\circ}$ was about 3 -fold as large as that in $0^{\circ}$, and those in $30^{\circ}$ and $60^{\circ}$ were approximately 2 -fold as large as that in $0^{\circ}$. In the same orientation, there was no significant difference to the standard deviations between the horizontal and vertical ref- ormations. The same tendency was also found in the visual evaluation. The pattern of the luminal artifact changed with the reconstruction direction. In the case of $90^{\circ}$ to the z-axis, band-like low attenuation artifacts were seen in the vertical reformation, whereas mesh-like low attenuation artifacts were seen in the horizontal reformation (Figs 6,7).

\section{Discussion}

Drug-eluting stents for coronary arteries have been developed and begun to be used clinically, ${ }^{12-14}$ so the opportunities to evaluate these stents using multidetector CT will increase. In the present study, we used Bx-Velocity stents because the Sirolimus-eluting Bx-Velocity (CYPHER, Johnson \& Johnson) is currently one of the most widely used drug-eluting stents! ${ }^{12,14}$

Many factors are thought to affect the accuracy of the evaluation of coronary stents by CT. Investigators have evaluated coronary stents using multidetector $\mathrm{CT}^{15-20}$ in which the image quality of the stented vessels depended on the patient's heart rate.17 Also, an increase of the CT attenuation inside the stent lumen and narrowing of the lumina were related to strut diameter and the metal content of the stent ${ }^{18}$ Convolution kernels with higher spatial resolution improve visualization of the coronary stent lumen and detection of in-stent stenoses 15,16 Hong et al reported the assessment of coronary stent patency based on measured contrast enhancement and showed that the stent diameter, convolution kernel and vessel orientation to the z-axis all affect the accuracy of the evaluation of coronary stents in phantoms?

In the present study of the luminal diameter measurement inside the stent, the absolute mean measurement error was significantly smaller with CC and CD. Improvement of artificial lumen narrowing using convolution kernels with higher spatial resolution has been reported ${ }^{15,16}$ However, even when we used these functions, the measured diameters of the stented lumen were approximately $45 \%$ and $35 \%$ smaller than the actual inner diameter of the 3- and 4-mm diameter models, respectively. Such artificial lumen narrowing may exert a significant practical impact on the estimation of stent restenosis. In an evaluation of 29 patients by Maintz et al, artificial lumen narrowing made it difficult to detect stenoses in 4 cases that showed low-grade stenosis on conventional angiography! ${ }^{17}$ The artificial lumen narrowing is attributed to the fact that the high $\mathrm{CT}$ attenuation of the stent struts raise the CT attenuation of the lumen near the stent on the profile curve. Therefore, evaluation of in- 
stent stenosis may become more difficult when the stent diameter is smaller. In contrast to our results, Hong et al reported that the error in the luminal diameter measurement inside the stent tended to increase as the actual diameter of the stent increased (they assessed 26 stents of 5 types in 19 patients) ${ }^{20}$ The differences in the type of the stents used and contrast enhancement among the patients may have affected their results.

When we used convolution kernels with higher spatial resolution, such as $\mathrm{CC}$ and $\mathrm{CD}$, it became easier to evaluate the stenosis inside the stents, which agrees with the findings of Maintz et al, who reported that a dedicated sharp kernel for image reconstruction facilitated improved visualization of the coronary artery stent lumen and detection of in-stent stenoses. 16 The slope of the profile curve corresponding to the boundary of the stent and the lumen or the boundary of the lumen and neointima is steeper with a convolution kernel emphasizing edge recognition, as shown in Fig 4. This is considered to be the reason that a convolution kernel with higher spatial resolution is suitable for the evaluation of the stent lumen. However, the noise and artifacts increase with these convolution kernels. Therefore, the convolution kernel with higher spatial resolution is not necessarily more suitable for stent evaluation. In our assessment, there was almost no difference between $\mathrm{CC}$ and $\mathrm{CD}$ in stent evaluation, although there were some differences in distinguishing severe stenosis and obstruction. Further assessment of convolution kernels is required, including developing a kernel for stent evaluation.

In our study, the luminal attenuation inside the patent stents of the models was higher than that of the unstented portions $(330 \mathrm{HU})$, which agrees with the in vivo findings of Hong et $\mathrm{al}^{20}$ who reported that the luminal attenuation inside the patent stent was higher than that of the contrastenhanced lumen proximal and distal to the stent. They suggested the possibility of assessing coronary stent patency on the basis of measured contrast enhancement. However, their study included only patent stents. In our results, the luminal attenuation inside the stent of the $50 \%$ stenosis model, as well as in the patent model, was higher than that of the unstented portion, although the luminal attenuation inside the stent of the $75 \%$ or $100 \%$ stenosis model was lower than that of the unstented portion (Fig 5). So, it will be impossible to distinguish stents with $50 \%$ stenosis from patent stents on the basis of measured contrast enhancement inside the stents. Significant stenosis will be indicated by lower luminal attenuation inside the stents than that in the unstented portion.

The in-stent artifact was the minimum in the case of $0^{\circ}$ to the $\mathrm{z}$-axis, and the maximum in the case of $90^{\circ}$. It was very difficult to evaluate the stent lumen at $90^{\circ}$ to the $\mathrm{z}$-axis, which has a significant practical impact on visual stent evaluation. A stent in the mid-portion of the right coronary artery, almost parallel to the $\mathrm{z}$-axis, is more easily evaluated than that in the proximal portion of the left anterior descending branch, almost orthogonal to the z-axis. This is considered to be because more of the stent struts exist in an $\mathrm{xy}$-plane as the vessel orientation approaches $90^{\circ}$.

The CT attenuation and the shape of the intimal hyperplasia and atheromatous plaque inside the stent will also affect accuracy. Some investigators have evaluated atherosclerotic plaque using $\mathrm{CT}^{21-26}$ and we simulated obstruction and stenosis by models with a density of approximately $80 \mathrm{HU}$, based on previously reported findings 21,22 The CT attenuation of non-calcified plaque is $76 \pm 35 \mathrm{HU}$ according to Becker et $\mathrm{al}^{21}$ whereas Estes et $\mathrm{al}^{22}$ reported that the mean $\mathrm{CT}$ attenuation of fibrous and lipid plaque is $90 \pm 24 \mathrm{HU}$ and $39 \pm 12 \mathrm{HU}$, respectively. Maintz et al made vascular models with stenosis using tubular wax cut into halves and implanted in the middle of the stent 16 We fixed a tube with a smaller diameter within a stent-mounted tube to simulate stenosis, because intimal hyperplasia tends to distribute concentrically inside the stent.

We fixed the vascular model within a cylinder filled with salad oil, because fat tissue usually exists around the vascular structures in humans. We fixed the vascular model in a water-filled spherical container of $12-\mathrm{cm}$ diameter to reduce the changes in the shape and area of the crosssection in an angulated position to the z-axis.

The acquisition parameters for CT scans in the present study were the same as the parameters used clinically, except for the tube current. We decreased the tube current intentionally because the phantom was smaller than a human body.

\section{Study Limitations}

First, we used only one kind of stent. Maintz et al reported that an increase of the CT attenuation inside the stent lumen and narrowing of the lumina depended on the strut diameter and metal content of the stent.$^{18}$ Because the stents used in our investigation had thick struts (strut diameter: $0.14 \mathrm{~mm}$ ), CT evaluation of them was considered to be comparatively difficult. Therefore, better results may be obtained when other stents with a smaller strut diameter are used. Second, we used static phantoms. Motion artifacts will affect visualization of the stent lumen in vivo, although improvement in the temporal resolution of CT scanners has decreased the effects of motion artifacts. ${ }^{19}$ Third, the obstruction and stenosis were simulated by models with a homogeneous density of approximately $80 \mathrm{HU}$, whereas in vivo intimal hyperplasia and atheromatous plaque are often heterogeneous. That may limit the extension of our findings to humans. Further studies are required to evaluate the effects of noise, and the density of intravascular contrast material on stent evaluation.

\section{Conclusion}

Visualization of stent lumen on CT is affected by the stent diameter, convolution kernel, and vessel orientation to the $\mathrm{z}$-axis, and these factors must be taken into consideration in the stent evaluation.

\section{Acknowledgments}

We thank Daisuke Suzuki for the visual estimation of the stent lumen, Fumiaki Harada and Takeshi Kawai for their advice and expertise, Terumo Co, Ltd, for generously providing the tubes made of ethylenevinyl alcohol copolymer and measuring the vascular models with a profile projector, and Johnson \& Johnson for generously providing the stents and information about their products.

\section{References}

1. Ropers D, Baum U, Pohle K, Anders K, Ulzheimer S, Ohnesorge B, et al. Detection of coronary artery stenoses with thin-slice multidetector row spiral computed tomography and multiplanar reconstruction. Circulation 2003; 107: 664-666.

2. Achenbach S, Giesler T, Ropers D, Ulzheimer S, Anders K, Wenkel $\mathrm{E}$, et al. Comparison of image quality in contrast-enhanced coronaryartery visualization by electron beam tomography and retrospectively electrocardiogram-gated multislice spiral computed tomography. Invest Radiol 2003; 38: 119-128.

3. Mohlenkamp S, Pump H, Baumgart D, Haude M, Gronemeyer DH, 
Seibel RM, et al. Minimally invasive evaluation of coronary stents with electron beam computed tomography: In vivo and in vitro experience. Cathet Cardiovasc Interv 1999; 48: 39-47.

4. Pump H, Mohlenkamp S, Sehnert CA, Schimpf SS, Schmidt A, Erbel R, et al. Coronary arterial stent patency: Assessment with electron-beam CT. Radiology 2000; 214: 447-452.

5. Pump H, Moehlenkamp S, Sehnert C, Schimpf SS, Erbel R, Seibel $\mathrm{RM}$, et al. Electron-beam CT in the noninvasive assessment of coronary stent patency. Acad Radiol 1998; 5: 858-862.

6. Yamaoka O, Ikeno K, Fujioka H, Kinoshita M, Murata K, Morita R, et al. Detection of Palmaz-Schatz stent by ultrafast CT. J Comput Assist Tomogr 1995; 19: 128-130.

7. Nieman K, Ligthart JM, Serruys PW, de Feyter PJ. Images in cardiovascular medicine: Left main rapamycin-coated stent: Invasive versus noninvasive angiographic follow-up. Circulation 2002; 105: e130e131.

8. Funabashi N, Komiyama N, Komuro I. Patency of coronary artery lumen surrounded by metallic stent evaluated by three dimensional volume rendering images using ECG-gated multislice computed tomography. Heart 2003; 89: 388.

9. Shaohong Z, Yongkang N, Zulong C, Hong Z, Li Y. Images in cardiovascular medicine: Imaging of coronary stent by multislice helical computed tomography. Circulation 2002; 106: 637-638.

10. Funabashi N, Komiyama N, Yanagawa N, Mayama T, Yoshida K, Komuro I. Images in cardiovascular medicine. Coronary artery patency after metallic stent implantation evaluated by multislice computed tomography. Circulation 2003; 107: 147-148.

11. Schuijf JD, Bax JJ, Jukema JW, Lamb HJ, Warda HM, Vliegen HW, et al. Feasibility of assessment of coronary stent patency using 16slice computed tomography. Am J Cardiol 2004; 94: 427-430.

12. Regar E, Serruys PW, Bode C, Holubarsch C, Guermonprez JL, Wijns W, et al; RAVEL Study Group. Angiographic findings of the multicenter Randomized Study With the Sirolimus-Eluting Bx Velocity Balloon-Expandable Stent (RAVEL): Sirolimus-eluting stents inhibit restenosis irrespective of the vessel size. Circulation 2002; 106: 1949-1956.

13. Grube E, Silber S, Hauptmann KE, Mueller R, Buellesfeld L, Gerckens U, et al. TAXUS I: Six- and twelve-month results from a randomized, double-blind trial on a slow-release paclitaxel-eluting stent for de novo coronary lesions. Circulation 2003; 107: 38-42.

14. Moussa I, Leon MB, Baim DS, O’Neill WW, Popma JJ, Buchbinder $\mathrm{M}$, et al. Impact of sirolimus-eluting stents on outcome in diabetic patients: A SIRIUS (SIRolImUS-coated Bx Velocity balloon-expandable stent in the treatment of patients with de novo coronary artery lesions) substudy. Circulation 2004; 109: 2273-2278.

15. Mahnken AH, Buecker A, Wildberger JE, Ruebben A, Stanzel S, Vogt F, et al. Coronary artery stents in multislice computed tomography: In vitro artifact evaluation. Invest Radiol 2004; 39: 27-33.

16. Maintz D, Seifarth H, Flohr T, Kramer S, Wichter T, Heindel W, et al. Improved coronary artery stent visualization and in-stent stenosis detection using 16-slice computed-tomography and dedicated image reconstruction technique. Invest Radiol 2003; 38: 790-795.

17. Maintz D, Grude M, Fallenberg EM, Heindel W, Fischbach R. Assessment of coronary arterial stents by multislice-CT angiography. Acta Radiol 2003; 44: 597-603.

18. Maintz D, Juergens KU, Wichter T, Grude M, Heindel W, Fischbach R. Imaging of coronary artery stents using multislice computed tomography: In vitro evaluation. Eur Radiol 2003; 13: 830-835.

19. Kruger S, Mahnken AH, Sinha AM, Borghans A, Dedden K, Hoffmann R, et al. Multislice spiral computed tomography for the detection of coronary stent restenosis and patency. Int J Cardiol 2003; 89: 167-172.

20. Hong C, Chrysant GS, Woodard PK, Bae KT. Coronary artery stent patency assessed with in-stent contrast enhancement measured at multi-detector row CT angiography: Initial experience. Radiology 2004; 233: 286-291.

21. Becker CR, Nikolaou K, Muders M, Babaryka G, Crispin A, Schoepf UJ, et al. Ex vivo coronary atherosclerotic plaque characterization with multi-detector-row CT. Eur Radiol 2003; 13: 2094-2098.

22. Estes JM, Quist WC, Lo Gerfo FW, Costello P. Noninvasive characterization of plaque morphology using helical computed tomography. J Cardiovasc Surg 1998; 39: 527-534.

23. Inoue $\mathrm{F}$, Sato $\mathrm{Y}$, Matsumoto N, Tani S, Uchiyama T. Evaluation of plaque texture by means of multislice computed tomography in patients with acute coronary syndrome and stable angina. Circ J 2004; 68: $840-844$.

24. Imazeki T, Sato $\mathrm{Y}$, Inoue $\mathrm{F}$, Anazawa T, Tani S, Matsumoto N, et al. Evaluation of coronary artery remodeling in patients with acute coronary syndrome and stable angina by multislice computed tomography. Circ J 2004; 68: 1045-1050.

25. Sato Y, Imazeki T, Inoue F, Yoshimura A, Fukui T, Horie T, et al. Detection of atherosclerotic coronary artery plaques by multislice computed tomography in patients with acute coronary syndrome: Report of 2 cases. Circ J $2004 ; 68: 263-266$.

26. Komatsu S, Hirayama A, Omori Y, Ueda Y, Mizote I, Fujisawa Y, et al. Detection of coronary plaque by computed tomography with a novel plaque analysis system, 'Plaque Map', and comparison with intravascular ultrasound and angioscopy. Circ J 2005; 69: 72-77. 